\title{
Genetic diversity of the honeybee Apis cerana in Yunnan, China, based on mitochondrial DNA
}

\author{
L. Yin and T. Ji \\ College of Animal Science and Technology, Yangzhou University, \\ Yangzhou, China \\ Corresponding author: $\mathrm{T}$. Ji \\ E-mail: jiting12@sohu.com
}

Genet. Mol. Res. 12 (2): 2002-2009 (2013)

Received January 23, 2013

Accepted May 15, 2013

Published June 20, 2013

DOI http://dx.doi.org/10.4238/2013.June.20.1

\begin{abstract}
DNA sequence diversity in the tRNA ${ }^{\text {leu }}$-COII portion of the mitochondrial genome was investigated in samples of Apis cerana from Yunnan, China. A fragment of about $480 \mathrm{bp}$ in tRNA ${ }^{\mathrm{leu}}$-COII, including a noncoding area and part of COII, was sequenced. The noncoding area was $97-98 \mathrm{bp} ; 8$ haplotypes were found, among which 5 had been reported previously, while 3 were new. The mean diversity of haplotypes was $0.752 \pm 0.030(0.378-0.698)$, and nucleotide diversity was $0.01073 \pm 0.00087(0.00412-0.01123)$. A neighbor-joining tree was constructed based on 73 sequences of noncoding intergenic regions in the mtDNA of A. cerana from China and other Asian countries; all haplotypes found in China fell into the mainland Asian group. This result does not support the hypothesis that $A$. cerana indica occurs in southern Yunnan, which was concluded in a recent report based on morphological variation.
\end{abstract}

Key words: Apis cerana; Genetic diversity; Genetic differentiation; mtDNA 


\section{INTRODUCTION}

Yunnan has always been known as the "Treasure House of Biodiversity" in China. There are many different species, which are distributed from tropical to arctic areas and have a wide range of genetic variation. There are also the richest bee species resources in Yunnan, and 6 of 9 known honeybee species are found distributed in Yunnan Province (Li et al., 1986). Among the 5 honeybee species occurring naturally in this province (Apis cerana, A. florea, A. andreniformis, A. labriosa, and A. dorsata), only A. cerana is of interest for commercial beekeeping because it can be maintained in hives, like $A$. mellifera (Wongsiri et al., 1986). In particular, $A$. cerana is widely distributed throughout the various geographic regions and climatic zones of Yunnan (Cheng, 1993), and traditional A. cerana beekeeping is rooted in the history of the region. Nevertheless, the levels of genetic diversity and population subdivision of $A$. cerana in Yunnan have not been well studied. This basic information is important for understanding distribution patterns and colonization of this species. Some studies have been conducted just using morphological markers (Kuang and Li, 1990; Tan et al., 2003, 2008; Li et al., 2012), whereas some research has just focused on small samples in Yunnan (Tan et al., 2006, 2007). To provide more data on this species, we carried out a survey of the tRNA ${ }^{\text {leu }}$-COII portion of the mitochondrial genome in Chinese A. cerana in Yunnan. mtDNA strictly abides by the rule of maternal inheritance in genetic processes and the mtDNA of one matriarchal ancestors' progeny is the same, so the mtDNA type of an individual can represent a matriarchy. That is to say, only a small number of samples can reflect the genetic structure of the population, so the number of animals for experiments can be greatly reduced. Because of the special reproductive mechanism of bees, a swarm of all the workers have the same mtDNA as the queen, and just one worker can represent the whole colony, which makes mtDNA a more convenient and effective tool to study genetic resources.

The objectives of this study were to estimate the level of genetic variability and population differentiation of $A$. cerana in Yunnan using sequences of mtDNA gene segments. This useful information can be applied for more effective natural resource management and conservation of $A$. cerana in Yunnan, and provides new data for study on the classification of A. cerana.

\section{MATERIAL AND METHODS}

\section{Sample collection}

Samples of A. cerana were collected from 6 locations in Yunnan (Table 1); a total of 60 colonies were sampled. Workers were collected from managed, semi-managed and wild colonies. The samples were frozen and brought to the laboratory in vials containing $100 \%$ ethanol. DNA was extracted from the worker's head according to the method reported by Ji et al. (2005).

\section{mtDNA sequencing}

The tRNA ${ }^{\text {leu}}-\mathrm{COII}$ region of the mitochondrial genome was amplified using primers designed by Cornuet et al. (1991): E2: 5'-GGC AAG AAT AAG TGC ATT G-3' and H2: 5'- 
CAA TAT CAT TGA TGA CC-3'. Polymerase chain reaction (PCR) was performed in a final volume of $20 \mu \mathrm{L} 1 \mathrm{X}$ PCR buffer, $0.2 \mathrm{mM}$ each dNTP, $400 \mathrm{nM}$ each primer, $40 \mathrm{ng}$ total DNA, and $0.15 \mathrm{U}$ Taq polymerase (Takara, Japan). DNA was amplified (Eppendorf Mastercycler Pro Gradient PCR Instrument, Germany) using the following program: an initial denaturation for 3 min at $96^{\circ} \mathrm{C}, 45 \mathrm{~s}$ annealing at $50^{\circ} \mathrm{C}$, and a 2 -min extension at $70^{\circ} \mathrm{C}$, repeated for 35 cycles. The PCR products were sent to Beijing Dingguo Changsheng Biotechnology Co., Ltd., for sequencing. Sequences were manually aligned with previously published sequences.

\begin{tabular}{|c|c|c|c|c|}
\hline Name (abbreviation) & Number of samples & Collecting locacity & $\begin{array}{l}\text { Geographic latitude } \\
\text { and longitude }\end{array}$ & Altitude $/ \mathrm{m}$ \\
\hline Apis cerana from Tengchong (TC) & 10 & Tengchong, Yunnan & $\begin{array}{l}25^{\circ} 01^{\prime} \mathrm{N}, \\
98^{\circ} 29^{\prime} \mathrm{E}\end{array}$ & 1700 \\
\hline A. cerana from Wuliangshan (WLS) & 10 & Wuliangshan, Yunnan & $\begin{array}{l}24^{\circ} 26^{\prime} \mathrm{N} \\
100^{\circ} 41^{\prime} \mathrm{E}\end{array}$ & 2100 \\
\hline A. cerana from Diqing (DQ) & 10 & Diqing, Yunnan & $\begin{array}{l}27^{\circ} 40^{\prime} \mathrm{N} \\
99^{\circ} 55^{\prime} \mathrm{E}\end{array}$ & 3400 \\
\hline A. cerana from Wuding (WD) & 10 & Wuding, Yunnan & $\begin{array}{l}25^{\circ} 50^{\prime} \mathrm{N} \\
102^{\circ} 15^{\prime} \mathrm{E}\end{array}$ & 1895 \\
\hline A. cerana from Lushui (LS) & 10 & Lushui, Yunnan & $\begin{array}{l}25^{\circ} 59^{\prime} \mathrm{N} \\
98^{\circ} 49^{\prime} \mathrm{E}\end{array}$ & 1800 \\
\hline A. cerana from Xishuangbanna (XSBN) & 10 & Xishuangbanna, Yunnan & $\begin{array}{l}21^{\circ} 6^{\prime} \mathrm{N} \\
100^{\circ} 07^{\prime} \mathrm{E}\end{array}$ & 1105 \\
\hline
\end{tabular}

\section{Statistical analyses}

Sequences were aligned using the LI-COR Align IR and compared with reported haplotypes from the NCBI (http://www.ncbi.nlm.nih.gov/). Haplotype diversity and nucleotide diversity $(\pi)$ (Nei, 1987; Nei and Miller, 1990) were calculated using the DnaSP4.0 program (Rozas et al., 2003). Haplotype diversity considers the number and frequency of observed haplotypes, while $\pi$ considers sequence divergence between haplotypes as well as the frequency of each haplotype to estimate the average number of nucleotide differences per site between 2 sequences in a population. Because we consider insertion/deletion events as well as base substitutions to be valid characters, alignment gaps were replaced with "dummy bases", so they would be included in the calculations. Molecular evolutionary phylogenetic trees were constructed using MEGA3.1 (Kumar et al., 2004) by the neighbor-joining method (NJ) using a Kimura 2-parameter distance, and bootstrap was performed using 1000 replicates; population differentiation $\left(F_{\mathrm{ST}}\right)$ was calculated using the Arlequin 3.0 software (Excoffier et al., 2005).

\section{RESULTS}

\section{Genetic diversity}

Eight haplotypes were found among the samples, 5 (Yunnan 1, 3, and 5-7) have been previously reported, while 3 (Yunnan 2, 4, and 8) were new. All 9 sequences were $96-98$ bases (Figure 1). 


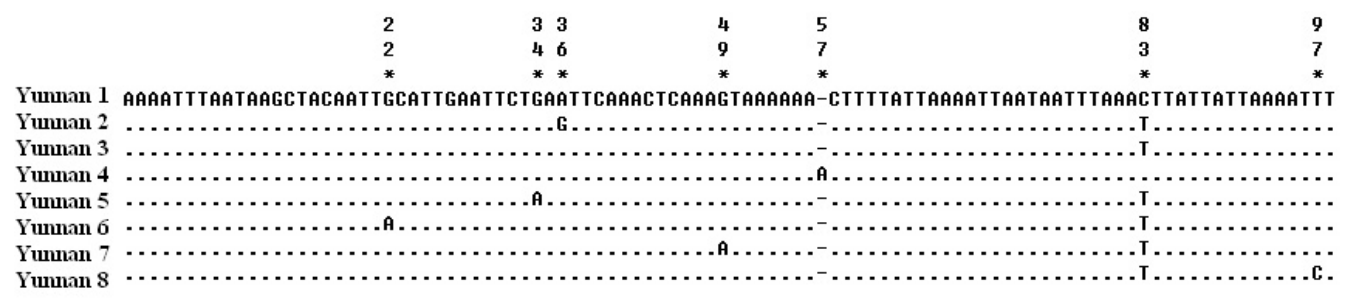

Figure 1. Sequences of the noncoding region of Apis cerana found in Yunnan. Numbers (vertica 1) show the variation positions among haplotypes. Dots indicate the nucleotides identical to the Yunnan 1 haplotype. Gaps are shown by dashes.

The geographic distribution of the haplotypes is shown in Table 2. Among the 8 haplotypes, Yunnan 3, which has been found in Japan, was the most common across all of our samples, except the Tengchong (TC) population, making up 21 of the 60 sequences. Yunnan 1 was a striking geographic pattern in Yunnan both in our research and a previous study (GenBank accession No. DQ388609.1), and also the major haplotype (18/60, 30\%). Yunnan 5-7 have also been found elsewhere in mainland China. The new Yunnan 2 was distributed in TC and Xishuangbanna (XSBN), and the new Yunnan 4 and Yunnan 8 were only distributed in Wuliangshan (WLS) and Wuding (WD), respectively.

\section{Table 2. Distribution of mtDNA noncoding area haplotypes of 6 populations.}

\begin{tabular}{|c|c|c|c|c|c|c|}
\hline \multirow[b]{2}{*}{ Haplotypes } & \multicolumn{6}{|c|}{ Populations } \\
\hline & $\mathrm{TC}$ & WLS & DQ & WD & LS & XSBN \\
\hline Yunnan 1 & 6 & 2 & & & 7 & 3 \\
\hline Yunnan 2 & 4 & & & & & 5 \\
\hline Yunnan 3 & & 7 & 8 & 1 & 3 & 2 \\
\hline Yunnan 4 & & 1 & & & & \\
\hline Yunnan 5 & & & 1 & 6 & & \\
\hline Yunnan 6 & & & 1 & & & \\
\hline Yunnan 7 & & & & 1 & & \\
\hline Yunnan 8 & & & & 2 & & \\
\hline
\end{tabular}

For abbreviations, see Table 1.

Haplotype diversity among our samples was $0.752 \pm 0.030$ and $\pi$ was $0.01073 \pm$ 0.00087 (Table 3), which reflected the mtDNA diversity of $A$. cerana in Yunnan. Although the overall haplotype diversity was high, the 6 populations showed differences: the lowest was for Diqing (DQ) at 0.378, and XSBN was the most abundant, 0.689.

Table 3. Haplotype diversity and nucleotide diversity of mtDNA noncoding area in 6 populations.

\begin{tabular}{|c|c|c|c|c|}
\hline Populations & Number of haplotypes & $\begin{array}{l}\text { Haplotype diversity } \\
\quad(\text { means } \pm \text { SD })\end{array}$ & Number of variable sites & $\begin{array}{l}\text { Nucleotide diversity } \\
\quad(\text { means } \pm \text { SD })\end{array}$ \\
\hline $\mathrm{TC}$ & 2 & $0.533 \pm 0.095$ & 2 & $0.01100 \pm 0.00195$ \\
\hline WLS & 3 & $0.467 \pm 0.132$ & 2 & $0.00481 \pm 0.00136$ \\
\hline DQ & 3 & $0.378 \pm 0.181$ & 2 & $0.00412 \pm 0.00212$ \\
\hline WD & 4 & $0.644 \pm 0.152$ & 3 & $0.01123 \pm 0.00726$ \\
\hline LS & 2 & $0.467 \pm 0.132$ & 1 & $0.00481 \pm 0.00136$ \\
\hline XSBN & 3 & $0.689 \pm 0.104$ & 2 & $0.01054 \pm 0.00169$ \\
\hline Total & 8 & $0.752 \pm 0.030$ & 7 & $0.01073 \pm 0.00087$ \\
\hline
\end{tabular}

For abbreviations, see Table 1. 


\section{Genetic differentiation}

Analysis of molecular variance (AMOVA) of mtDNA noncoding area in 6 A. cerana populations (Table 4$)$ resulted in a fixation index $\left(F_{\mathrm{ST}}\right)$ of $0.31723(\mathrm{P}<0.01)$, which indicated that the genetic differentiation of populations in Yunnan was significant.

Table 4. AMOVA of mtDNA noncoding area in 6 populations.
\begin{tabular}{lrcccc}
\hline Source of variation & d.f. & Sum of squares & Variance components & Percentage of variation & $F_{\text {ST }}$ \\
\hline Among populations & 5 & 10.5 & $1.20929(\mathrm{Va})$ & 23.83 & $0.31723^{* * * *}$ \\
Within populations & 54 & 21.2 & $3.86590(\mathrm{Vb})$ & 76.17 & \\
Total & 59 & 31.7 & 5.07519 & & \\
\hline
\end{tabular}

d.f. = degrees of freedom; $\mathrm{Va}=$ variance components among populations; $\mathrm{Vb}=$ variance components within populations; ***P $<0.01$.

Table 5 shows the pairwise $F_{\mathrm{ST}}$ (below matrix) and corresponding $F_{\mathrm{ST}} \mathrm{P}$ values (above matrix) for AMOVA. The $F_{\mathrm{ST}}$ value indicated that the genetic divergences of the WD population and other populations (except DQ) were significant or very significant $(\mathrm{P}<0.05$ or $\mathrm{P}<0.01)$.

Table 5. Paiwise $F_{\text {ST }}\left(\right.$ down matrix) and corresponding $F_{\text {ST }}$ P values (above matrix) for AMOVA.
\begin{tabular}{lcccccc} 
Population & TC & WLS & DQ & WD & LS & XSBN \\
\hline TC & - & 0.08108 & 0.00901 & 0.00000 & 0.09009 & 0.33333 \\
WLS & 0.16667 & - & 0.18919 & 0.00000 & 0.18018 & 0.10811 \\
DQ & 0.38889 & 0.1111 & - & 0.02703 & 0.00000 & 0.00901 \\
WD & 0.43275 & 0.32479 & 0.24036 & - & 0.00000 & 0.00000 \\
LS & 0.10853 & 0.16667 & 0.51852 & 0.51389 & - & 0.02703 \\
XSBN & 0.00427 & 0.17211 & 0.28889 & 0.37908 & 0.31070 & - \\
\hline
\end{tabular}

For abbreviations, see Table 1.

\section{Phylogenetic relationships}

The NJ phylogenetic dendrograms (Figure 2) suggested that 6 A. cerana populations in Yunnan could be subdivided into 2 groups, 1 from low altitude subtropical regions and another from high altitude temperate zone regions. An NJ tree based on 73 sequences of noncoding intergenic regions in mtDNA of $A$. cerana in China and other Asian countries was constructed (Figure 3). Haplotypes in China (excluding Yunnan) and other Asian countries are from Smith et al. (2000) and NCBI. The results showed that all haplotypes found in China fell into the mainland Asia group and were mixed with haplotypes found in the other Asian countries.

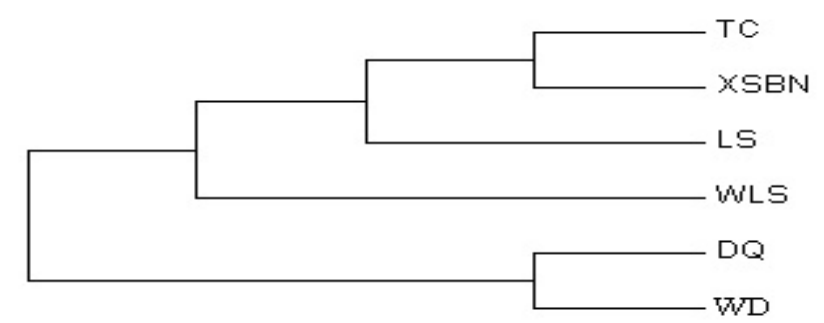

Figure 2. Neighbor-joining tree of mtDNA noncoding area of 6 A. cerana populations. $\mathrm{TC}=$ Tengchong; $\mathrm{XSBN}=$ Xishuangbanna; $\mathrm{LS}=$ Lushui; $\mathrm{WLS}=$ Wuliangshan; $\mathrm{DQ}=$ Diqing; $\mathrm{WD}=$ Wuding. 


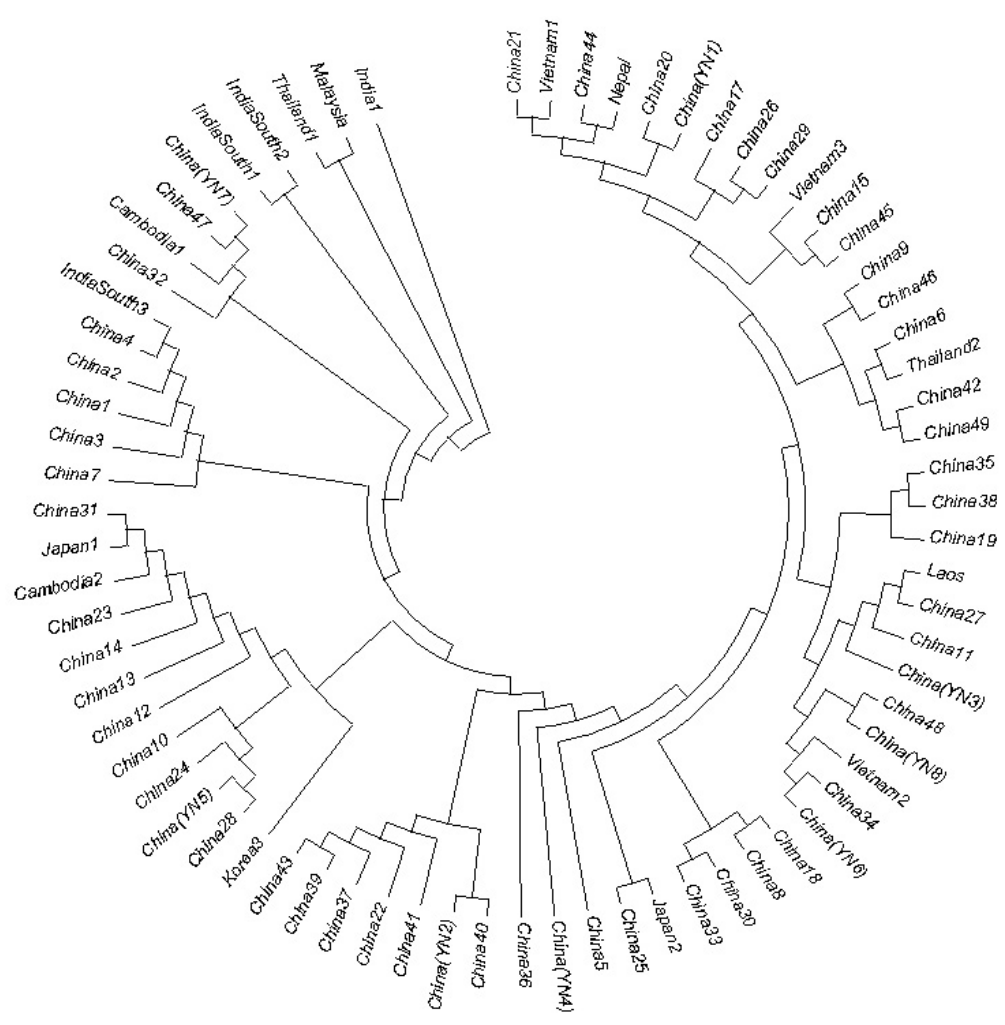

Figure 3. Neiboghr-joining tree based on the sequences of 73 noncoding intergenic regions in the mtDNA of Apis cerana in China and other Asia countries. Haplotypes in China (excluding Yunnan) and other Asia countries are from Smith et al. (2000) and NCBI.

\section{DISCUSSION}

\section{Genetic diversity and differentiation}

The overall haplotype diversity of $A$. cerana in Yunnan was found to be high, but the 6 populations showed differences: DQ was the lowest and XSBN was the most abundant. XSBN has a tropical monsoon climate, with plenty of sunshine, abundant rainfall and lush forest, which is a heaven for bees, so there are many wild eastern honeybees distributed here. The XSBN honeybees used in this research were all wild, and the XSBN population showed relatively abundant genetic diversity here. On the contrary, DQ is located in the northwest of Yunnan Province and in the Hengduan Mountain area, with cold temperature, complex terrain, and lack of nectar and pollen plants, and it is not the ideal place for eastern honeybees perching and reproducing; Yunling and $\mathrm{Nu}$ Mountains reduce genetic exchanges with other local eastern honeybees. All of these factors could account for the low genetic diversity of the DQ population. Darvill (2006) reported that a segregated population shows low genetic diversity. Low genetic diversity may lead to the homozygous sex allele and production of diploid 
drones, influence colony group's potential development, and affect the swarm survival ability, so the DQ population should be given more attention in future studies.

AMOVA of mtDNA noncoding area in $6 \mathrm{~A}$. cerana populations indicated that the genetic variation was significant within populations in Yunnan, and the genetic divergences of the WD population and other populations (except DQ) were significant or very significant. WD bees also had the most haplotypes and high haplotype diversity, so we can infer that WD bees had a complex genetic basis.

\section{Phylogenetic relationships}

The NJ phylogenetic dendrograms suggested that 6 A. cerana populations in Yunnan could be subdivided into 2 groups, XSBN was in the low altitude subtropical group, while DQ was in the high one. These results were supported by morphological variation (Tan et al., 2003). However, the other 4 populations were not strictly reallocated into these 2 groups, like with morphological variation. It is reasonable to speculate on the basis of the results that the mitochondrial data, which are not directly affected by natural selection, provide information on biogeographic patterns resulting from mutation, migration, and genetic drift, while morphological characters respond to selective pressures imposed by environmental conditions.

The subdivision of $A$. cerana into different subspecies and relationships to the known subspecies of the East Asian mainland is still controversial in China (Yang and Xu, 1982, 1986; Tan et al., 2006; Li et al., 2012). Smith and Hagen (1996) and Smith et al. (2000) examined the biogeography of $A$. cerana, and found 4 major groups of haplotypes: an Asian mainland, Sundaland, Palawan, and Luzon-Mindanao. However, their research lacked samples of China. To make up for the regret, we compared Chinese halotypes from GenBank and our research findings to other halotypes in the Asian mainland (Smith and Hagen, 1996; Smith et al., 2000; GenBank) (Figure 3). The results strongly supported the reports by Smith et al. (2000). However, it appears that the variation within this mitochondrial region is insufficient to fully resolve phylogeographic relationships within mainland China.

The subspecies of $A$. cerana in Yunnan is one of the hotspots in the study of $A$. cerana in China. Yang and Xu (1982) separated A. cerana in China into 5 different subspecies, corresponding to the regions of Hailan, Eastern Yunnan, Southern Yunnan, Aba, and Xizhang (Tibet), and they described 7 biotypes, which included the palm forest and mountain biotype of Hailan and other Asian countries and the biotypes of Guangdong-Guangxi, Hunan, Yunnan Plateau, Northern, and Changbeishan (Yang and Xu, 1986). Peng et al. (1989) reported that the South Yunnan bees belonged to $A$. c. indica, and Tan et al. (2006) supported this viewpoint on the basis of morphometry and ND2 analysis. However, our data do not yet appear to be comprehensive enough to support or negate the conclusions, because none of the halotypes found in Yunnan in our research fell together with the clusters of $A$. $c$. indica. It is likely that more samples and analyses with compatible methods are needed to resolve this problem.

\section{ACKNOWLEDGMENTS}

Research supported by the Special Fund for Modern Bee Industry and the Technology System provided by the China Agriculture Ministry (grant \#NYCYTX-43). We thank the Department of Agriculture and Forestry for providing honeybee samples. 


\section{REFERENCES}

Cheng YC (1993). Apiculture in China. China Agriculture Press, Beijing.

Cornuet JM, Garney L and Solignac M (1991). Putative origin and function of the intergenic region between COI and COII of Apis mellifera L. mitochondorial DNA. Genetics 128: 393-403.

Darvill B, Ellis JS, Lye GC and Goulson D (2006). Population structure and inbreeding in a rare and declining bumblebee, Bombus muscorum (Hymenoptera: Apidae). Mol. Ecol. 15: 601-611.

Excoffier L, Laval G and Schneider S (2005). Arlequin (version 3.0): An integrated software package for population genetics data analysis. Evol. Bioinform. Online 1: 47-50.

Ji T, Chen J and Pan R (2005). Compare of bee's genome DNA concentration by different abstracting method. Apic. China 12: $10-11$.

Kuang BY and Li YQ (1990). Study on subspecies of Apis cerana Fabricius in Yunnan, China. J. Yunnan Agric. Univ. 5: 224-229.

Kumar S, Tamura K and Nei M (2004). MEGA3: Integrated software for Molecular Evolutionary Genetics Analysis and sequence alignment. Brief. Bioinform. 5: 150-163.

Li H, Hu ZW, Wang ZW, Zhuang D, et al. (2012). Morphologic study on Apis cerana in middle and northwest of Yunnan province. J. Yunnan Agric. Univ. 4: 611-615.

Li SW, Meng YP, Chang JT, Li JH, et al. (1986). A comparative study of esterase isozymes in 6 species of Apis and 9 genera of Apoidea. J. Apic. Res. 25: 129-133.

Nei M (1987). Molecular Evolutionary Genetics. Columbia University Press, New York.

Nei M and Miller JC (1990). A simple method for estimating average number of nucleotide substitutions within and between populations from restriction data. Genetics 125: 873-879.

Peng YS, Nasr ME and Locke SJ (1989). Geographical races of Apis cerana Fabricius in China and their distribution. Review of recent Chinese publications and a preliminary statistical analysis. Apidologie 20: 9-20.

Rozas J, Sánchez-DelBarrio JC, Messeguer X and Rozas R (2003). DnaSP, DNA polymorphism analyses by the coalescent and other methods. Bioinformatics 19: 2496-2497.

Smith DR and Hagen RH (1996). The biogeography of Apis cerana as revealed by mitochondrial DNA sequence data. $J$. Kansas Entomol. Soc. 64: 294-310.

Smith DR, Villafuerte L, Otis G and Palmer MR (2000). Biogeography of Apis cerana F. and A. nigrocincta Smith: insights from mtDNA studies. Apidologie 31: 265-279.

Tan K, Fuchs S, Koeniger N and Zan RG (2003). Morphological characterization of Apis cerana in the Yunnan Province of China. Apidologie 34: 553-561.

Tan K, Meixner MD, Fuchs S, Zhang X, et al. (2006). Geographic distribution of the eastern honeybee, Apis cerana (Hymenoptera: Apidae), across ecological zones in China: Morphological and molecular analyses. Syst. Biodivers. 4: 473-482.

Tan K, Warrit N and Smith DR (2007). Mitochondrial DNA diversity of Chinese Apis cerana. Apidologie 38: 238-246.

Tan K, Hepburn HR, Radloff SE, Fuchs S, et al. (2008). Multivariate morphometric analysis of the Apis cerana of China. Apidologie 39: 343-353.

Wongsiri S, Lai YS and Liu ZS (1986). Beekeeping in the Guangdong Province of China and some observations on the Chinese honey bee, Apis cerana cerana, and the European honey bee, Apis mellifera ligustica. Am. Bee J. 126: 748752.

Yang GH and Xu SY (1982). The distribution and geographical variation of Apis cerana sinensis in China. China Beekeeping 3: 17-19.

Yang GH and Xu SY (1986). Geographic distribution of Apis cerana Fabr. and their subspecies diversity. J. Yunnan Agric. Univ. 1: 86-92. 